\title{
Aportes de la psicología organizacional y del trabajo en Colombia a los sistemas de gestión de la calidad modelo ISO $9001^{*}$
}

\author{
Contributions of working and organizational psychology \\ in Colombia to quality management systems ISO 9001 model
}

Recibido: 21 de enero de 2013

Revisado: 23 de julio de 2013

Aceptado: 15 de agosto de 2013

\author{
Javier Giraldo** \\ Convenio Universidad Santo Tomás e ICONTEC
}

\section{RESUMEN}

Este estudio es producto de una investigación cualitativa hermenéutica que buscó comprender los aportes de la psicología organizacional y del trabajo (POT) a la conceptualización e instrumentalización de los sistemas de gestión de la calidad (SGC) modelo ISO 9001, acudiendo a la búsqueda de términos relativos a la gestión y principios (categorías deductivas) contemplados en la NTC ISO 9000. La investigación se realizó mediante la consulta de 23 revistas colombianas de psicología indexadas (desde 1992 a 2012), por medio del análisis de contenido y la interpretación de frases con sentido de 86 artículos pertinentes en relación con las temáticas aquí abordadas. Se halló que la POT hace referencia frecuentemente a los términos relativos a la organización (y dentro de esta al ambiente de trabajo) y a la calidad (en la cual

\footnotetext{
* Artículo de investigación.

** Correspondencia: Javier Vicente Giraldo Jaramillo. Especialista en Administración: Gerencia de Recursos Humanos, especialista en Administración y Gerencia de Sistemas de la Calidad. Docente de la Facultad de Psicología, Universidad Santo Tomás (Bogotá). Correo electrónico: jvgiraldo@ hotmail.com.
}

Trabajo de grado para optar al título de magíster en Calidad y Gestión Integral, dirigido por Mg. Christian Mora Bustos. 
predomina el concepto de "competencia"). Esto, a su vez, se relaciona con los temas presentes en la POT (categorías inductivas), identidad (los SGC como constructos sociales que median, en gran medida, lo identitario y la calidad de vida de las personas y la sociedad) y el clima organizacional -(léase en los SGC como ambiente de trabajo). Se reconocen aportes de la POT en lo conceptual, instrumental y crítico a los SGC modelo ISO 9001. Igualmente, se hace evidente la brecha, ya que desde la POT se concibe a los SGC modelo ISO 9001 como un tema instrumental y cuantitativo positivista, de ahíla baja sinergia entre estos, lo cual revierte en la dificultad para la contextualización y el cumplimiento de su responsabilidad social.

Palabras clave: sistemas de gestión de la calidad, psicología organizacional y del trabajo (POT), hermenéutica.

\section{ABSTRACT}

This study is the result of a hermeneutic qualitative research, which try to understand the contribution of the Work and Organizational Psychology (WOP) to the and conceptualization and tools of the Quality Management System (QMS), version 9001, using terms related to management and to principles (deductive categories) of NTC ISO 9000. This research was done consulting 23 Colombian magazines related to psychology (since 1992 to 2012), throughout content analysis and phrases interpretation with sense of 86 relevant articles to the topic of this one. It was found that WOP frequently makes reference to the terms related to organization (including work environment) and to quality (including competence as predominant term). This relate too with the subjects include in WOP (inductive categories), identity (the QMS as social constructs which are between the identity and the life quality of people and society) and organizational environment -read in the QMS like work environment-. It is recognize the WOP contributions to the conceptual, instrumental and critics for the QMS version 9001. Equally, it is evident the gap considering that WOP conceive QMS version 9001 as a instrumental and quantitative theme - positivity, which explain the low synergy between those ones, and as a consequence cause the difficulty for the contextualization and accomplishment of its social responsibility.

Keywords: Quality management systems, work and organizational psychology (WOP), hermeneutic.

\section{INTRODUCGIÓN}

El objetivo de la investigación fue comprender el aporte de la psicología organizacional y del trabajo (POT) a los sistemas de gestión de la calidad (SGC) modelo ISO 9001, mediante la comprensión de los términos relativos a la gestión y principios de la calidad de la ISO 9000, presentes en los artículos de las revistas indexadas de psicología en Colombia, en el período comprendido entre 1992 y 2012, con el fin de dar cuenta de la relación entre los elementos investigados.

Estos temas (POT y SGC modelo ISO 9001), de gran relevancia en el mundo globalizado y en la construcción identitaria individual, organizacional y social, tienen una gran responsabilidad social. Por un lado, como ciencia, el progreso de la psicología está determinado por la pertinencia y oportunidad con que asuma las demandas sociales. Esto implica que, además de proveer conocimientos, se constituye en la base para que se apliquen dichos conocimientos y se solucionen los problemas prácticos mediante la tecnología. 
De otro lado, se encuentra la calidad, que la ISO $9000^{1}$, define como "grado en el que un conjunto de características (...) inherentes cumple con los requisitos", entendiendo que "Inherente (...) significa que existe en algo, especialmente como una característica permanente" y que por requisito se entiende la "Necesidad 0 expectativa establecida, generalmente implícita u obligatoria". Así, "la familia de Normas ISO 9000 (...) se han elaborado para asistir a las organizaciones, (...), en la implementación y la operación de sistemas de gestión de la calidad eficaces", ya que "para conducir y operar una organización en forma exitosa se requiere que esta se dirija y controle en forma sistemática y transparente. Se puede lograr el éxito implementando y manteniendo un sistema de gestión que esté diseñado para mejorar continuamente su desempeño mediante la consideración de las necesidades de todas las partes interesadas"3.

Con todo, si se tiene en cuenta que la organización es objeto de los SGC, de acuerdo con la ISO 9000, es un "conjunto de personas (...) con una disposición de responsabilidades, autoridades y relaciones", acogiendo en calidad de parte interesada a la "persona o grupo que tenga un interés en el desempeño o éxito de una organización”. Se admite que el trabajador se realiza con esta y que como ser social y único tiene identidad con ella. De este modo, acorde con Castel $^{6}$, no se debe olvidar que el trabajo como

1 INSTITUTO COLOMBIANO DE NORMAS TÉCNICAS Y CERTIFICACIÓN (ICONTEC). Sistema de gestión de la calidad. Fundamentos y vocabulario. NTC-ISO 9000:2000. Numerales 3.1.1 y 3.1.2, Bogotá, D. C.: ICONTEC, 2005. p. 18.

2 Ibíd, p. 8.

3 Ibíd, p. 8.

4 Ibíd, 3.3.6, p. 22.

5 Ibíd, 3.3.7, p. 22.

6 CASTEL, Robert. (2000). As transformações da Questão Sócial. En: Desigualdade e a Questão Social. São Paulo: EDUC. p. 235-264. actividad transformadora es cada vez más relevante en la vida, como organizador social y referente de inclusión.

Sin embargo, el ambiente de trabajo, entendido como "conjunto de condiciones bajo las cuales se realiza el trabajo", que incluye factores "(...) sociales, psicológicos (...)", entre otros, en organizaciones que asumen este sistema, frecuentemente es inadecuado. González expresa que “(...) no es difícil encontrar que son varios los casos en que la norma ISO dejó de ser un valor agregado al sistema de mejoramiento de la calidad, convirtiéndose en un serio problema que afecta el ambiente organizacional (...)"'; consecuente con esto "no es raro encontrarse con empresas certificadas que adhirieron a la cultura de la ineficiencia (...)"10, esto a pesar de que "con la globalización comercial se depende cada vez más de las normas internacionales por la creciente interdependencia y exigencia de los mercados"11.

Se podría afirmar, con Rentería, que la serie de normas ISO 9000 no pasan de ser retórica:

(...) las personas continúan siendo 'vistas' o 'asumidas' en la práctica como cualquiera de los otros recursos (...), podría decirse que (...), exigencias como las de certificación (...) tipificadas por la estandarización, mantienen la vigencia de los modelos de administración científica soportados en metáforas como la de la 'máquina' (...), lo que constituye una paradoja para los sistemas estratégicos dependientes de la polivalencia y la especialidad

7 ICONTEC. Op. cit., 3.3.4, p. 22.

8 Ibíd. Nota. 3.3.4, p. 22

9 GONZÁLEZ, Tito. Dificultades en la certificación de calidad normas ISO. Buenos Aires: El Cid, 2009. p. 3. 27 p.

10 MÉNDEZ, José Claudio; JARAMILLO, David y SERRANO, Ildefonso. Gestión de la calidad en procesos de servicios y productivos. México, D. F.: Instituto Politécnico Nacional, 2009. p. $9.236 \mathrm{p}$.

11 ICONTEC. Op. cit., p. 20. 
esperada de las personas en función de formas de adaptación y mantenimiento o no de su trabajo, y su aporte a la efectividad organizacional $1^{12}$.

Como lo expresa Morin, es una "carrera ciega: (...) Porque la tragedia del desarrollo y el subdesarrollo del desarrollo, la desenfrenada carrera de la tecnociencia, la ceguera que produce el pensamiento parcelario y reductor, todo ello nos ha arrojado a una descontrolada aventura" 13 .

El problema se acentúa, ya que, conforme lo expresa Peralta:

(...) parece existir un vacío desde la Psicología Organizacional, por falta de referentes para entender el mundo del trabajo y los sujetos que en él se constituyen. Este vacío se nota en la poca comprensión que muestran los psicólogos organizacionales de las vivencias, experiencias y formas de sentido que otorgan los trabajadores (...) a su situación actual. Existen pocos escritos e investigaciones sobre el tema $(\ldots)^{14}$.

Paradójicamente, "sea como fuere, la disciplina psicológica sigue su marcha expansiva dentro del mundo del trabajo, aunque adolezca de las dificultades que se señalan, pues se parte de la suposición generalizada de que el conocimiento psicológico opera produciendo aumentos en la productividad y mejoras en el

12 RENTERÍA, Érico. De recursos humanos a la psicología organizacional y del trabajo: reflexiones a la luz de las realidades actuales del mundo del trabajo. p. 27-51. En: AGUILAR, María Constanza y RENTERÍA, Érico (Comp.). Psicología del trabajo y de las organizaciones. Reflexiones y experiencias de investigación. Bogotá, D. C.: Universidad Santo Tomás y Universidad del Valle, 2009. p. $42-43$.

13 MORÍN, Edgar. La agonía planetaria. En: Revista Colombiana de Psicología. 1994, n. 3 3 p. 33, p. 29-34. Consultado el 29 de agosto de 2012. Disponible en Internet: http://www.revistas.unal.edu.co/ index.php/psicologia/article/viewFile/15810/16640.

14 PERALTA, María Claudia. La construcción de sujetos en las nuevas realidades laborales: reflexiones para su estudio desde la psicología organizacional y del trabajo. En: p. 53-74. En: AGUILAR y RENTERÍA. Op. cit., p. 57-58. bienestar de los trabajadores, y por ende contribuye a la prosperidad general de la sociedad"15.

Se observan también deficiencias de la psicología como ciencia, ya que de acuerdo con Leser y Souza, Estamos diante de um quadro gravissimo e inaceitável, nà o só porque há psicólogos vitimando pessoas, mas tambén porque a credibilidade de uma ciência e profissào que conta com excelentes pesquisadores e profissionais, capazes de contribuir para a construçào da cidadania, está ameaçada ${ }^{16}$.

En consonancia, Braginsky y Braginskyrefieren objeciones a la POT:

(...) la falta de unidad de la disciplina, la falta de dirección, la pretensión de emulación de las ciencias naturales, la incapacidad para investigar fenómenos significativos de manera significativa, la falta de relevancia para el mundo de la vida y la falta de una visión que haga justicia a la realidad humana ${ }^{17}$.

Básicamente es la misma crítica que hace Rose a lo que denomina "la calidad en la práctica", ya que el valorar los sistemas desde su desempeño "(...) puede ser oscurecido por la arrogancia, la ignorancia o la parcialidad" 18 .

Paradójicamente se encuentra que, al igual que con los SGC ISO 9001, la WOP (Work and Organizational

15 PULIDO, Hernán Camilo. Examinando las operaciones de la psicología en su relación con el trabajo. En: AGUILAR y RENTERÍA. Op. cit., p. 81 .

16 LESER, Sylvia y SOUZA, María Helena. Psicologia da Violência ou Violência da Psicologia. En: Psicologia USP (Universidade de São Paulo). 2008, vol. 19, n. ${ }^{\circ}$ 4, p. 593.

17 BRAGINSKY, Benjamin y BRAGINSKY, Dorothea. 1974. p. 214. En: PULIDO, Hernán Camilo. En búsqueda de una psicología crítica en los ámbitos laborales. Universitas Psychologica. 2004, vol. 3, n. ${ }^{0}$, p. 214.

18 ROSE, Kenneth H. Gestión de calidad de proyectos. Qué, cómo y por qué. Bogotá: Panamericana, 2008. p. 189-208. 
Psychology) has had a long and important history in the Colombian psychology and has played a key role in the development of psychology in Colombia, (... $)^{19}$.

En este sentido, pareciera que la POT y los SGC ISO 9001 se hubiesen permeado por este oscuro panorama y no estuvieran respondiendo a las exigencias que les demanda su ser. Antes bien, sirven para legitimar fenómenos como la exclusión y la desigualdad, en los que la lógica económica y el interés particular priman: “(...) el sistema de desigualdad reposa paradójicamente en el esencialismo de la igualdad, (...) en la cientifización de la normalidad, (...)" ${ }^{20}$.

A pesar lo manifestado, de acuerdo con Enciso y Perilla, el psicólogo organizacional “(...) cada vez cobra un papel más protagónico como socio estratégico de las organizaciones que quieren lograr mayor competitividad (...)"21. Agregan que "Colombia, (...) se encuentra todavía en un nivel muy básico de modernización socioempresarial (...), ya que aún la mayoría (de las empresas) opera bajo la inecuación de máxima producción a mínimos costos en todos los factores, incluyendo el humano"'22.

Con todo, es necesario investigar la relación existente entre los SGC ISO 9001 y la POT, con el fin de

19 AGUILAR, María Constanza, LÓPEZ, Wilson, GAMBOA, Juan Pablo. Present and Future of Work and Organizational Psychology in Colombia. 2008. En: International Association of Applied Psychology (IAAP). Division 1. Work and Organizational Psychology. Newsletter. p. 11. Consultado el 10 de abril de 2009. Disponible en Internet: http://www.iaapsy.org/division1/uploads/ newsletter/May \%2020081.pdf.

20 DE SOUSA, Adriana. Cambio organizacional. Bogotá: Asociación Colombiana para el Avance de las Ciencias del Comportamiento (ABA) Colombia, 2003. p. 127. Consultado el 20 de abril de 2012. Disponible en Internet: www.abacolombia.org.co.

21 ENCISO, Eleonora y PERILLA, Lyria Esperanza. Visión retrospectiva, actual y prospectiva de la psicología organizacional. En: Acta Colombiana de Psicología. 2004, n.o 11, p. 5-22.

22 Ibíd, p. 6. entrever la sinergia y/o la brecha entre estos dos vitales de la contemporaneidad, del desarrollo de la identidad y realización del ser humano, y la sostenibilidad y competitividad organizacional.

La POT debe ejercer un papel líder acorde con la realidad organizacional. Para hacerlo, es necesario investigar cómo la norma ISO 9001 aporta a los SGC. Se desaprovechan cúmulos de información disponible. Esta relación sinérgica es necesaria, ya que como dice Schvarstein: "Es preciso que las organizaciones ejerzan su responsabilidad social que se les reclama y que algunas de ellas declaman (...). Han de ser organizaciones socialmente inteligentes, y será esta inteligencia una condición de existencia (...) necesaria para que puedan operar" ${ }^{\prime 23}$.

\section{METODOLOGÍA}

La investigación respondió al paradigma - interpretativo-comprensivo (hermenéutica). Lo interpretativo guía los procesos de construcción del conocimento.

El objeto de estudio se constituye como texto de sentido, ya que "(...) es la estructura semántica o textual de la actividad práctica cotidiana”" ${ }^{24}$. No se estudia directamente, sino desde las comprensiones que se han realizado de él, en este caso, desde categorías y principios como construcciones de los sujetos. Por ello, en esta investigación se realizó un acercamiento detallado de cada uno de los textos en un contexto específico

23 SCHAVARSTEIN, Leonardo. La inteligencia social de las organizaciones. Buenos Aires: Paidós, 2003. p. 45.

24 PACKER, Martín. La investigación hermenéutica en el estudio de la conducta humana. En: Psicología Cultural. Grupo Cultura \& Desarrollo Humano, 25 p. Traducción de American Psychologist, 1985, vol. 40, n. ${ }^{\circ} 10$, p. 11-12. Consultado el 10 de enero de 2013. Disponible en Internet: http://psicologiacultural.org/Pdfs/ Traducciones/La\%20investigacion\%20hermeneutica.pdf. 
y, a partir de ello, se construyó una versión sobre el fenómeno.

Consecuente con lo mencionado, la presente investigación usó un enfoque de tipo cualitativo. Esta manera de investigar privilegia la profundidad sobre la extensión. Estudia detalladamente un fenómeno en un contexto determinado, bajo el supuesto de que el estudiarlo en contextos concretos permite evidenciar más detalles sobre el fenómeno, como es el caso de esta investigación.

De acuerdo con Vasilachis, El interés fundamental radica en la forma en que el "(...) mundo es comprendido, experimentado, producido" ${ }^{25}$. En este caso, se averigua cómo los diferentes autores están comprendiendo el fenómeno de los SGC ISO 9001 desde la POT en Colombia.

El interprete, sujeto que se enfrenta al texto, según Cárcamo ${ }^{26}$, parte de un horizonte de sentido diferente del texto: lee desde un horizonte que lo lleva a acercarse al texto desde ciertas intencionalidades y sentidos. Sin intérprete no se puede considerar que haya texto.

La relación texto e intérprete se genera en la comprensión. Esta se da a través de un proceso constante, que Martínez ${ }^{27}$ define como natural, porque los humanos siempre conocen de manera hermenéutica, buscando los sentidos. Sostiene, además, que el proceso de búsqueda de sentido implica comprender en

25 VASILACHIS, Irene. La investigación cualitativa. En: VASILACHIS (Coord.). Estrategias de investigación cualitativa. Barcelona: Gedisa, 2006. p. 27 y p. 23-64.

26 CÁRCAMO, Héctor. Hermenéutica y análisis cualitativo. En: Cinta de Moebio. 2005, n. ${ }^{0}$ 23, p. 1-14. Consultado el 18 de noviembre de 2012. Disponible en Internet: http://redalyc.uaemex. $\mathrm{mx} /$ src/inici o/ArtPdfRed.jsp?iCve=10102306.

27 MARTÍNEZ, Miguel. Comportamiento humano. Nuevos métodos de investigación. México: Trillas, 2002.307 p. función de una "parte", pero también en función de un "todo" que se constituye como su contexto; es un continuo ir y venir entre texto, contexto e intérprete, en interacción dialéctica. En el caso de la presente investigación, el proceso comprensivo se inicia en las partes, consideradas "unidades de análisis"; luego se entienden en función del texto y se vuelve a las partes como tal.

Ahora bien, desde la hermenéutica, y en esta investigación específicamente, se reconoce que el acto comprensivo está mediado por el horizonte de sentido del intérprete. Así, Gadamer ${ }^{28}$ considera que es imposible el conocimiento totalmente objetivo, por ello el hermeneuta debe dar cuenta de sus juicios. El horizonte de sentido de esta investigación estuvo constituido por los "términos relativos a la gestión" (numeral 3.2 categorías y subcategorías) de la NTC-ISO $9000^{29}$, de tal forma que, acudiendo a los "principios de la gestión de la calidad" de esta misma norma, desde allí se constituyen las interpretaciones. El acto comprensivo estuvo dado por la investigación.

Con todo, es lícito concluir con Bautista que "la hermenéutica debe ser vista como una forma de comprensión crítica" ${ }^{30}$. Así, de acuerdo con Martínez ${ }^{31}$, en los estudios hermenéuticos se define la validez contextual.

Para la selección de los artículos se recurrió al "(...) muestreo teórico que se realiza para descubrir las categorías y sus propiedades, y para sugerir las

28 GADAMER, Hans-Georg. En: ECHEVERRÍA, Rafael. El búho de Minerva. Santiago de Chile: Comunicaciones Noreste Ltda., 2004. p. 122.

29 ICONTEC. Op. cit., numeral 2.

30 BAUTISTA, Nelly Patricia. Proceso de investigación cualitativa. México: Manual Moderno, 2011. p. 50.

31 MARTÍNEZ. Op. cit., p. 33. 
interrelaciones (...)"32. Se tuvieron en cuenta los criterios de pertinencia temática, ubicación, disponibilidad y rigurosidad de producción. Para asegurar el criterio de indexación se recurrió al listado de revistas colombianas de psicología de ASCOFAPSI ${ }^{33}$.

Finalmente, a partir de los criterios mencionados, de 42 revistas se seleccionaron $23(54,8 \%)$, revisando las publicaciones desde 1992 a 2012, para escoger 86 artículos.

Como ya se expresó, la técnica "unidad de análisis" para los textos se constituye en la oración con sentido, es decir, se analizaron expresiones de los textos referidas a una idea principal. Esta "unidad de análisis" asegura la comprensión del texto (oración con sentido) en su contexto (párrafo y artículo).

Como instrumento se usaron las matrices descriptiva e interpretativa. La matriz descriptiva contiene la referencia bibliográfica y el criterio de selección de cada uno de los artículos seleccionados. De otro lado, la información consignada en la matriz interpretativa es de carácter literal, esto para asegurar la rigurosidad en la recolección de la información. En esta matriz, a partir de la comprensión del artículo, del párrafo y de las categorías, se realizó la interpretación desde los principios, ya que "Estos ocho principios de gestión de la calidad constituyen la base de las normas de sistemas de gestión de la calidad de la familia de normas ISO $9000 " 34$.

32 GLASER, Barney y STRAUSS, Anselm. The Discovery of Grounded Theory: Strategies for Qualitative Research. Chicago: Aldine Publishing Company, 1999. p. 67.

33 ASCOFAPSI (Asociación Colombiana de Facultades de Psicología). Listado de revistas y publicaciones de psicología producidas en Colombia (23 de julio de 2012). En: Observatorio de Calidad de la Educación Superior en Psicología. p. 1-44. Consultado el 27 de agosto de 2012. Disponible en Internet: http://www.ascofapsi.org. co/documentos/2009/revistas_psicologia_colombia. pdf.

34 ICONTEC. Op. cit., p. i-ii.
Acogiendo a Martínez ${ }^{35}$, la investigación se desarrolló en tres etapas:

\section{De construcción de la investigación. Esta tuvo} como objetivo preparar el escenario investigativo en términos de problema, marcos y métodos. Aquí se desarrollan las siguientes actividades: se determinaron el objeto de estudio, sus límites y alcances; se seleccionaron las fuentes y, por último, se eligieron los instrumentos y procedimientos de aplicación.

2. Interpretativa y de sistematización. En esta fase, la información se sistematizó mediante el análisis de contenido de tipo categorial, de acuerdo con los presupuestos formulados por López ${ }^{36}$, Piñue $^{37}$ y Rodríguez ${ }^{38}$. Incluyó:

\section{a. Establecimiento de categorías, de acuerdo} con los referentes ISO 9000. Se definió la unidad de análisis: oración con sentido. Estas hacen referencia, de manera explícita o implícita, al concepto de calidad y/o a los SGC modelo ISO 9001, a los principios y/o a los términos relativos a la gestión.

b. Categorización. Se procedió a realizar este proceso en cada oración con sentido y en cada

35 MARTÍNEZ. Op. cit., p. 37.

36 LÓPEZ, Fernando. El análisis de contenido como método de investigación. En: XXI Revista de Educación. 2002, n. ${ }^{\circ}$ 4, p. 167-179. Consultado el 12 de julio de 2013. Disponible en Internet: http:// uhu.es/publicaciones/ojs/index.php/xxi/article/viewFile/610/932.

37 PIÑUEL, José Luis. Epistemología, metodología y técnicas del análisis de contenido. En: Estudios de Sociolingüistica. 2002, vol. 3, n. ${ }^{0}$ 1, p. 1-42. Consultado el 12 de julio de 2013. Disponible en Internet: http://pendientedemigracion.ucm.es/info/mdcs/A. Contenido.pdf.

38 RODRÍGUEZ, Yajaira. La hermenéutica aplicada a la interpretación del texto. El uso de la técnica de contenido. En: Revista Ciencias de la Educación. 2002, vol. 2, n.o 2, p. 143-154. Consultado el 31 de octubre de 2012. Disponible en Internet: http://servicio. bc.uc.edu.ve/educacion/revista/a2n20/2-20-8.pdf. 
artículo, lo cual implicó su lectura y posterior segmentación. Cada uno de los segmentos se transcribió en la matriz y luego se categorizó. Después, con el fin de no perder la unidad del texto, de cada segmento, se realizó la interpretación de cada artículo y se ubicaron los principios a los que estos apuntaban, además del aporte conceptual, instrumental o crítico del artículo como un todo que responde a una lógica y a un propósito. Igualmente, de este proceso surgieron categorías inductivas.

c. Validación de la categorización. Todo ello fue revisado por tres expertos competentes, quienes revisaron la definición de las categorías y el proceso mismo de categorización, previa contextualización con la investigación. En esta revisión de las interpretaciones realizadas, de acuerdo con Rodríguez"(...) se adopta el principio de la inmanencia de validez de la teoría en su base más que en su expresión empírica, (...) toca los frentes de coherencia, comprenhensibilidad, congruencia discursiva, compenetración de horizontes en el análisis de los textos y prospectividad" 39 .

\section{Etapa de divulgación. Esta incluyó:}

a. Organización de interpretaciones. Las interpretaciones se analizan como un todo, con el fin de dar cuenta de las tendencias, de las categorías deductivas y las inductivas; solo así se vislumbran los elementos de brecha y cercanía entre la POT y los SGC ISO 9001.

\section{b. Generación de estructura del documento final. Corresponde a la entrega para evaluación de jurado, lo que Cortés ${ }^{40}$ denomina "amigo crítico". Esto hizo más confiable el proceso.}

c. Construcción del informe final. Una vez aprobado por el jurado, se comparte y se juzga la calidad de la investigación, a modo de otro "amigo crítico".

Todo esto se constituye con criterios de calidad que permiten evaluar el "resultado" y dan cuenta de la rigurosidad del "proceso". En este sentido, se acudió a los expuestos por Mendizábal ${ }^{41}$ como ejes transversales: credibilidad, transferibilidad, seguridad, confirmabilidad y empoderamiento.
40 CORTÉS, Graciela. Confiabilidad y validez en estudios cualitativos. En: Educación y Ciencia. 1997, vol. 1, n.o 1, p. 77-82. Consultado el 12 de julio de 2013. Disponible en Internet: http:// educacionyciencia.org/index.php/educacionyciencia/article/view $/ 111$.

41 MENDIZÁBAL, Nora. Los componentes del diseño flexible en la investigación cualitativa. En: VASILACHIS. Op. cit. p. 65-105. 
RESULTADOS Y DISCUSIÓN

Como se puede observar en la tabla 1 , predominan los términos relativos a la organización, calidad y gestión (94\%). Los relativos al proceso y producto, examen, y gestión de la calidad para los procesos de medición y auditoría apenas ocupan el $6 \%$, mientras que los relacionados con las características, documentación y conformidad cobran el $0 \%$.

Tabla 1. Frecuencia de uso de los "términos relativos a la gestión" (categorías) de la NTC-ISO 9001 en la POT

\begin{tabular}{|l|c|c|}
\hline \multicolumn{1}{|c|}{ Categoría } & Frecuencia & $\%$ \\
\hline $\begin{array}{l}\text { Términos relativos a la } \\
\text { organización }\end{array}$ & 72 & 34 \\
\hline Términos relativos a la calidad & 62 & 30 \\
\hline Términos relativos a la gestión & 62 & 30 \\
\hline $\begin{array}{l}\text { Términos relativos al proceso } \\
\text { yal producto }\end{array}$ & 4 & 2 \\
\hline $\begin{array}{l}\text { Términos relativos al examen } \\
\text { Términos relativos a la gestión } \\
\text { de la calidad para los procesos de } \\
\text { medición }\end{array}$ & 2 & 1 \\
\hline $\begin{array}{l}\text { Términos relativos a la auditoría } \\
\text { Términos relativos a las } \\
\text { características }\end{array}$ & 2 & 1 \\
\hline $\begin{array}{l}\text { Términos relativos a la } \\
\text { conforminidad }\end{array}$ & 0 & 0 \\
\hline
\end{tabular}

Fuente: elaboración propia.

Consistente con lo mencionado, las temáticas predominantes (subcategorías) desde la POT en la comprensión de los SGC modelo ISO 9001 se pueden observar en la tabla 2.
Tabla 2. Frecuencia de uso de los "términos relativos a la gestión" (subcategorías) de las normas

ISO 9000 en la POT

\begin{tabular}{|c|c|c|c|}
\hline Subcategoría & Categoría & Frecuencia & $\%$ \\
\hline Competencia & $\begin{array}{l}\text { Término relativo a } \\
\text { la calidad }\end{array}$ & 62 & 15,0 \\
\hline $\begin{array}{l}\text { Ambiente de } \\
\text { trabajo }\end{array}$ & $\begin{array}{l}\text { Término relativo a } \\
\text { la organización }\end{array}$ & 60 & 14,6 \\
\hline Sistema & $\begin{array}{l}\text { Término relativo a } \\
\text { la gestión }\end{array}$ & 36 & 8,7 \\
\hline Gestión & $\begin{array}{l}\text { Término relativo a } \\
\text { la gestión }\end{array}$ & 32 & 7,8 \\
\hline Calidad & $\begin{array}{l}\text { Término relativo a } \\
\text { la calidad }\end{array}$ & 30 & 7,3 \\
\hline Alta dirección & $\begin{array}{l}\text { Término relativo a } \\
\text { la gestión }\end{array}$ & 26 & 6,3 \\
\hline Organización & $\begin{array}{l}\text { Términos relativo } \\
\text { a la organización }\end{array}$ & 24 & 5,8 \\
\hline Eficiencia & $\begin{array}{l}\text { Término relativo a } \\
\text { la gestión }\end{array}$ & 22 & 5,3 \\
\hline Eficacia & $\begin{array}{l}\text { Término relativo a } \\
\text { la gestión }\end{array}$ & 18 & 4,4 \\
\hline Cliente & $\begin{array}{l}\text { Término relativo a } \\
\text { la organización }\end{array}$ & 16 & 3,9 \\
\hline Parte interesada & $\begin{array}{l}\text { Término relativo a } \\
\text { la organización }\end{array}$ & 14 & 3,4 \\
\hline $\begin{array}{l}\text { Estructura de la } \\
\text { organización }\end{array}$ & $\begin{array}{l}\text { Término relativo a } \\
\text { la organización }\end{array}$ & 12 & 2,9 \\
\hline \multirow[t]{2}{*}{$\begin{array}{l}\text { Satisfacción del } \\
\text { cliente }\end{array}$} & $\begin{array}{l}\text { Término relativo a } \\
\text { la calidad }\end{array}$ & 10 & 2,4 \\
\hline & & TOTAL & 87,9 \\
\hline
\end{tabular}

Fuente: elaboración propia.

Complementario a esto, se realizó la clasificación de los temas, tal como se tratan en la literatura POT referente a los SGC ISO 9001 (ver tabla 3).

Como se puede observar, lo relacionado con "competencia" y "ambiente de trabajo" es frecuente (29,6 \%), coincidiendo con dos temáticas de la POT, 
como son "competencias" y "clima organizacional, con $34 \%$ (Pereda y Berrocal ${ }^{42}$, Enciso ${ }^{43}$, Hay Group ${ }^{44}$, Ruiz, Jaraba y Romero ${ }^{45}$, Saravia ${ }^{46}$, Casallas ${ }^{47}$, etc.). Igualmente, resalta la "alta dirección" (6,3\%), que desde POT se asume como "liderazgo" (8\%), el cual presenta relevancia (Tejada ${ }^{48}$, Berger et al. ${ }^{49}$, ReyesJacome y Lara $^{50}$, etc.).

42 PEREDA, Santiago y BERROCAL, Francisca. Gestión de recursos humanos por competencias. Madrid: Centro de Estudios Ramón Areces, S. A., 1999. 276 p.

43 ENCISO, Eleonora. Identificación y desarrollo de competencias estratégicas en la implementación de un sistema de aseguramiento de la calidad: normas ISO 9000. En: Acta Colombiana de Psicología. 2004, n. ${ }^{0} 11$, p. 63-80. Consultado el 17 de octubre de 2012. Disponible en Internet: http:// regweb.ucatolica.edu.co/publicaciones/psicologia/ ACTA/n11/articulosrevista/art\%206\%20acta\%2011.pdf.

44 HAY GROUP. Las competencias: clave para una gestión integrada de los recursos humanos. Barcelona: Deusto, 2004. 189 p.

45 RUIZ, Maritza, JARABA, Brunoy ROMERO, Lidia. Competencias laborales y la formación universitaria. En: Psicología desde el Caribe. 2005, n ${ }^{0}$ 16, p. 64-91. Consultado el 15 de septiembre de 2012. Disponible en Internet: http://rcientificas.uninorte.edu.co/ index.php/psicologia/article/viewFile/ 1996/1281.

46 SARAVIA, Marcelo. Recursos humanos en el siglo XXI. Gestión de competencias laborales desde un modelo práctico. 2008. Consultado el 15 de abril de 2012. Disponible en Internet: http://www.conacyt. gov.bo/ convocatorias/publicaciones/GestionCompetencias Laborales.pdf.

47 CASALLAS, Walter Smith. Identificación de competencias laborales de asesores comerciales en una compañía de aseguramiento y prestación de servicios de salud. En: Avances en Psicología Latinoamericana. 2009, vol. 27, n. ${ }^{0} 1$, p. 207-230. Consultado el 1 de octubre de 2012. Disponible en Internet: http://revistas.urosario. edu.co/index.php/apl/article/view/5/6.

48 TEJADA, Fabio. El sistema de gestión de la calidad como aporte para el desarrollo de habilidades y capacidad gerencial en las pymes de Bogotá. En: Signos. Investigación en Sistemas de Gestión. 2009, vol. 1, n. ${ }^{\circ} 1$, p. 33-68.

49 BERGER, Rita, et al. Validity of the Human System Audit Transformational Leadership Short Scale (HSA-TFL) in Four European Countries. En: Universitas Psychologica. 2011, vol.10, n. ${ }^{0}$ 3, p. 657-668. Consultado el 26 de septiembre de 2012. Disponible en Internet: http://revistas.javeriana.edu.co/ind ex.php/revPsycho/ article/view/831/1162.

50 REYES-JÁCOME, Laura y LARA, Gustavo. El liderazgo integral en las organizaciones. En: Avances en Psicología Latinoamericana.
Tabla 3. Frecuencia de uso de las temáticas de la POT en la literatura referente a los SGC ISO 9001

\begin{tabular}{|l|c|c|}
\hline \multicolumn{1}{|c|}{ Tema } & Frecuencia & $\%$ \\
\hline Competencias & 18 & 21 \\
\hline Identidad & 12 & 14 \\
\hline Clima organizacional & 11 & 13 \\
\hline Gestión del cambio & 8 & 9 \\
\hline Liderazgo & 7 & 8 \\
\hline Cultura organizacional & 5 & 6 \\
\hline Riesgos psicosociales & 5 & 6 \\
\hline Motivación y compromiso & 3 & 4 \\
\hline Ética y valores & 3 & 4 \\
\hline $\begin{array}{l}\text { Otros: participación, aprendizaje } \\
\text { organizacional, gestión del } \\
\text { conocimiento, trabajo en equipo, } \\
\text { servicio, comunicación, etc. }\end{array}$ & 12 & 15 \\
\hline
\end{tabular}

Fuente: elaboración propia.

Términos como "sistema", "gestión" y "calidad" se asumen desde la POT como elementos de conceptualización (v. gr. sistema) que abarcan fenómenos específicos ( $v$ gr. gestión que comprende alta dirección y, por ende, el ejercicio del liderazgo) y/o la característica que se encuentra presente en todo el quehacer organizacional ( $v . g r$. calidad), y que se convierte en indicador y garantía de sostenibilidad y mejora.

Preocupa que temáticas muy sensibles, como innovación, toma de decisiones, control, medición y otras, claves para SGC ISO 9001, se encuentren mínimizadas en la POT. Estas temáticas, además de no ser abordadas con frecuencia por la POT, mucho menos se relacionan con los SGC.

2011, vol. 29, n. ${ }^{\circ}$ 1, p. 148-160. Consultado el 1 de octubre de 2012. Disponible en Internet: http://revistas.urosario.edu.co/index.php/ apl/article/vi ew/1641/1475. 
Asimismo, como resultado de la presente investigación se encuentra el uso de los "principios de gestión de la calidad" de los sistemas basados en las normas NTCISO 9001 (ver tabla 4).

Tabla 4. Frecuencia de uso de los "principios de gestión de la calidad" de la NTC-ISO 9000 en la POT

\begin{tabular}{|l|c|c|}
\hline \multicolumn{1}{|c|}{ Principio } & Frecuencia & $\%$ \\
\hline Participación del personal & 72 & 25,0 \\
\hline Enfoque de sistema para la gestión & 56 & 19,4 \\
\hline Liderazgo & 54 & 18,8 \\
\hline Enfoque al cliente & 32 & 11,1 \\
\hline $\begin{array}{l}\text { Enfoque basado en hechos para la } \\
\text { toma de decisión }\end{array}$ & 26 & 9,0 \\
\hline Enfoque basado en procesos & 22 & 7,6 \\
\hline $\begin{array}{l}\text { Mejora continua } \\
\text { Relaciones mutuamente beneficiosas } \\
\text { con el proveedor }\end{array}$ & 6 & 6,9 \\
\hline
\end{tabular}

Fuente: elaboración propia.

Resaltan la "participación del personal" (25\%), el "liderazgo" (18,8 \%) y el "enfoque al cliente" $(11,1 \%)$ como temáticas que la POT aporta al enriquecimiento y la comprensión de los SGC ISO 9001. El "enfoque de sistema para la gestión" (19,4 \%), como lo expresa el enunciado, se asume como concepto que posibilita integralidad en la comprensión de los fenómenos. De otro lado, con respecto al "enfoque basado en hechos para la toma decisión", el "enfoque basado en procesos" y la "mejora continua", son asumidas por la POT como estrategias, no como objetos de estudio que impliquen lo disciplinar; no se desconoce su importancia, sino que tienen perspectiva instrumental. El problema es grave cuando "(...) la 'calidad' se encuentra bajo el horizonte de una razón instrumental" ${ }^{1}$.

51 RODRÍGUEZ, Carolina et al. Aproximaciones al concepto de calidad desde la filosofía. p. 228. En: Revista de la Universidad de La Salle. 2009, n. ${ }^{\circ} 48$, p. 225-241.
Por último, en cuanto a las "relaciones mutuamente beneficiosas con el proveedor", su frecuencia de aparición es baja (2,1\%), tal vez porque la temática implicada se asume desde el "cliente" y/o porque otros desarrollos los asimilan (por ejemplo, la psicología económica y la del consumidor) y/o porque realmente no se le da relevancia a esta como se debiera, negando aparentemente lo social en lo organizacional y lo sistémico de esta realidad.

De otra parte, es interesante observar que de los 86 artículos seleccionados, 50 (58 \%) son de discusión teórica (revisión, ensayo, comentario, reseña) y 36 (42\%) corresponden a investigaciones (bibliométricas, hermeneúticas, correlacionales, estudios de caso), esto es, que aportan desde el punto de vista disciplinario y profesionalizante de la psicología a la comprensión de los SGC ISO 9001. En realidad, es un porcentaje bajo con respecto al universo de artículos sobre POT.

Así mismo, es curioso encontrar que de los 86 artículos revisados, 62 (72\%) no tratan el tema directamente, aunque fueron seleccionados porque su contenido mencionaba temas relativos a la gestión y/o a los principios promulgados en ISO 9000 . De estos 86 , solamente $24(28 \%)$ refieren explícitamente algún SGG y 12 (14\%) tratan acerca de los SGC ISO 9001. Cabe suponer que la POT desconoce los aportes de los SGC ISO 9001 como instrumento aunque, paradójicamente, los cuestiona. Así, se entendería que el tema "identidad" ocupe el segundo lugar (14\%) en la frecuencia de uso de las temáticas de POT en la literatura referente a los SGC ISO 9001 (ver tabla 4). Se considera que este es un elemento necesario en el área organizacional de la psicología y en la norma ISO 9001, ya que metaanaliza, reconoce y cuestiona el quehacer y la responsabilidad que le atañe a lo investigado.

Preocupa que se lleguen a concebir la POT y los SGC ISO 9001 como frecuentemente respondientes (no 
responsables), reactivos (no proactivos), problemáticos (no problematizadores), construcciones demagógicas o instrumentales que perpetúan el taylorismo, la desmotivación, la desigualdad y la pobreza en una sociedad inequitativa que se retroalimenta para mantener su statu quo (Morin ${ }^{52}$, Prilleltensky ${ }^{53}$, Enciso y Perilla ${ }^{54}$, Leser y Souza ${ }^{55}$, Robledo ${ }^{56}$, Peralta ${ }^{57}$, González ${ }^{58}$, Rentería ${ }^{59}$, etc.). Otra comprensión respecto a "identidad" se refiere al tema de la cultura, ampliamente estudiado y que en algunas ocasiones incluye clima organizacional (ambiente de trabajo) (Kriegel y Brandt ${ }^{60}$, Vega et al. ${ }^{61}$, Méndez ${ }^{62}$, Soracipa ${ }^{63}$, etc.).

52 MORÍN, Edgar. La agonía planetaria. En: Revista Colombiana de Psicología. 1994, n. ${ }^{0}$ 3, p. 33, p. 29-34. Consultado el 29 de agosto de 2012. Disponible en Internet: http://www.revistas.unal.edu.co/ index.php/ psicologia/article/viewFile/15810/16640.

53 PRILLELTENSKY, Isaac. The Morals and Politics of Psychology. Psychological Discourse and the Status Quo. Albany: State University of New York Press, 1994. 283 p.

54 ENCISO y PERILLA. Op. cit. p. 12.

55 LESER y SOUZA. Op. cit. p. 592.

56 ROBLEDO, Ángela María. Psicología y mundos laborales contemporáneos. En: Cuadernos de Psicología. 2007, vol. 3, n. o 2, p. 5-9.

57 PERALTA. Op. cit., p. 58.

58 GONZÁLEZ. Op. cit., p. 7.

59 RENTERÍA. Op. cit., p. 42.

60 KRIEGEL, Robert y BRANDT, David. De las vacas sagradas se hacen las mejores hamburguesas. Bogotá, D.C.: Norma, 2006. p. 4.

61 VEGA, Diana et al. Panorama sobre los estudios de clima organizacional en Bogotá, Colombia (1994-2005). En: Diversitas: Perspectivas en Psicología. 2006, vol. 2, n. ${ }^{0}$ 2, p. 329-349. Consultado el 29 de septiembre de 2012. Disponible en Internet: http://www.scielo.org.co/pdf/dpp/v2n2/v2n2a13.pdf.

62 MÉNDEZ, Carlos Eduardo. Así somos... ¿ ¿Y qué? Cuatro relatos de cultura en gestión empresarial. Bogotá, D.C El autor, 2009. 161 p.

63 SORACIPA, Nancy Yaneth. Definición de estrategias de transformación de la cultura organizacional en función de un sistema de gestión de calidad. En: Signos. Investigación en Sistemas de Gestión. 2009, vol. 1, n. ${ }^{0}$ 1, p. 139-151.

\section{CONCLUSIONES}

Se puede afirmar, sin lugar a dudas, que se logró el propósito de la presente investigación, enunciado al inicio del artículo, particular y consecuentemente en lo referente a los objetivos específicos:

- Objetivo específico 1. Develar las categorías y los principios relacionados con los SGC ISO 9001 en la producción de la POT.

El cumplimiento de este objetivo se puede observar claramente en las tablas 1, 2, 3 y 4, así como en la presentación en el apartado anterior del presente documento, lo que a su vez proviene de la matriz referente a la interpretación de todos y cada uno de los artículos analizados por unidades "frases con sentido". Resumiendo, dentro de los elementos estrechamente relacionados se encuentran los siguientes:

- En principios (ver tabla 4), predominan "participación del personal" (25\%), "enfoque de sistema para la gestión" (19,4\%), "liderazgo" $(18,8 \%)$ y "enfoque al cliente" $(11,1 \%)$. Representan el 83,3\%.

- En categorías (términos relativos a la gestión) (ver tabla 2), predominan lo relativo a la organización $(34 \%)$, calidad (30\%) y gestión (30\%). Representan el $94 \%$.

- En subcategorías (términos relativos a la gestión) -(ver tabla 2), predominan "competencia" $(15 \%)$ y "ambiente de trabajo" $(14,6 \%)$. Representan el 39,6\%, lo cual es alto teniendo en cuenta el gran número de términos considerados (84 en total), por lo cual se puede decir que solo el 2,4\% (2) representa 39,6\%.

- Complementario a esto se encuentra la tabla 4, que se refiere a las temáticas que desde la 
POT han surgido (categorías inductivas), relacionadas con los SGC ISO 9001. Son "competencias" (21\%), "identidad" (14\%), "clima organizacional" (13\%), "gestión del cambio" (9\%), "liderazgo"(8\%), "cultura organizacional" (6\%), "riesgos psicosociales" (6\%), "motivación y compromiso" (4\%) y "ética y valores" (4\%). Todo ello representa el $85 \%$ de lo encontrado, ayuda a entender y valida los hallazgos referentes a principios y términos relativos a la gestión de los SGC ISO 9001.

- Objetivo específico 2. Describir la brecha entre la producción de la POT y los SGC modelo ISO 9001. Se encuentra lo siguiente:

- En principios (ver tabla 4), el "enfoque basado en procesos" (7,6\%), la "mejora continua" $(6,9 \%)$ y las "relaciones mutuamente beneficiosas con el proveedor" (2,1\%) ocupan un segundo lugar con 16,6\%. Esto puede deberse a que los dos primeros comprenden técnicas y se refieren a medios instrumentales, lo que, dicho sea de paso, se le critica a mucho al profesional que se dedica a los SGC ISO 9001, ya que se concibe a los SGC basados en ISO 9001 como los debe olvidándose de la 9000, 9004, 19011 y de las normas complementarias.

En cuanto al otro aspecto, "relaciones con el proveedor", este elemento se encuentra inmerso en psicología del consumidor o económica, o dentro de temáticas de la POT, como comunicación, trabajo en equipo, satisfacción, compromiso y motivación, razón por la cual este principio no resaltó como debiera.

- En categorías (términos relativos a la gestión) (ver tabla 1), lo referente al proceso y al producto $(2 \%)$, examen (2\%), gestión de la calidad para los procesos de medición (1\%) y auditoría
(1\%) ocupan el $6 \%$. De otra parte, los relativos a la auditoría, características, documentación y conformidad ocupan $0 \%$. Esto es consistente con el déficit detectado respecto a los principios, y ayuda a entender los elementos pertenecientes a esta.

- En subcategorías (relativas a la gestión) (ver tabla 3), "organización" (5,8\%), "eficiencia" $(5,3 \%)$, "eficacia" $(4,4 \%)$, "cliente" $(3,9 \%)$, "parte interesada" (3,4\%), "estructura de la organización" (2,9\%) y "satisfacción del cliente" $(2,4 \%)$ ocupan $28,1 \%$, sin contar otros que aparecen ocasionalmente 0 no aparecen. Se debe recordar que en total son 84, y que en la tabla 3 solo se registran 13 , que comprenden el $87,9 \%$. Esto reafirma lo expresado: pareciera a veces que lo organizacional y contextual propiamente dicho no interesaran, aunque sean categorías necesarias en la comprensión del comportamiento humano en la organización. Es una paradoja para la POT, dado el promulgado enfoque sistémico.

- Reafirmando lo anterior, se encuentra la tabla 3, que se refiere a las temáticas que desde la POT han surgido (categorías inductivas), relacionadas con los SGC ISO 9001, donde elementos como participación, aprendizaje organizacional, gestión del conocimiento, trabajo en equipo, servicio, comunicación, entre otros, ocupan todos el $15 \%$.

- Objetivo específico 3: generar aportes conceptuales y procedimentales desde la POT a los SGC modelo ISO 9001.

Los 86 artículos revisados representan una nutrida y rigurosa fuente de insumos para quienes trabajan en lo relacionado con los SGC. 
- Así, en cuanto a la construcción conceptual desde la POT, se hallaron 43 artículos (50\%). Estos superan el pragmatismo y el taylorismo. Además, el aporte de lo cualitativo y los modelos mixtos de investigación permite vislumbrar horizontes optimistas.

- En cuanto a lo procedimental, se encuentran 30 artículos (34,9\%). Estos, al provenir de la rigurosidad científica y con el filtro de la ética, permiten actuar de manera más responsable, sistémica y sistemática.

- Adicional a lo conceptual y a lo procedimental se encuentran los artículos (13-15,1\%) que tratan lo crítico, social e identitario. Estas categorías emergentes permiten metaposicionarse en el quehacer, entender el sentido, y autoevaluar la ética y la responsabilidad en el uso de los SGC ISO 9001.

\section{RECOMENDACIONES}

En cuanto a la temática abordada, se formulan las siguientes sugerencias:

- Realizar esta investigación teniendo en cuenta los discursos de las organizaciones y personas que han implementado SGC ISO 9001, o tienen la intención de hacerlo, para contrastar lo investigativo con la realidad empresarial y anclar estas dos temáticas en la sociedad actual.
- Acorde con lo mencionado, se sugieren variaciones por culturas, empresas, grado de desarrollo de los SGC ISO 9001, otros SGC, etc., lo cual permite generar aplicaciones contextualizadas respecto del fenómeno investigado.

De otra parte, en cuanto al método, que es novedoso y brinda una nueva perspectiva, se debe considerar lo siguiente:

- El análisis de contenido debe tomarse como una técnica flexible que permite llevar a cabo la investigación desde procesamientos matemáticos hasta cualitativos y hermenéuticos marcados. Se sugiere el uso de un software que facilite el análisis cuantitativo y cualitativo de datos textuales, por ejemplo, DataMining, SPAD-T, STATISTICA Text Miner, DB2 Intelligent Minerfor Text, QSR NUD*IST -Non Numerical Unstructed Data Indexing Searching and Theorizing-.

- Acudir a fuentes impresas (v. gr. libros de investigación) que, aunque no tienen igual impacto, visibilidad y uso en bases de datos, sí recogen textos científicos.

Acorde con lo mencionado, se deduce el cumplimiento de los objetivos propuestos, a la vez que esto sugiere rutas y desafíos. De otro lado, el uso en los medios académicos permite confrontar el discurso con otros "amigos críticos" y circularlo para su uso. Estos "nuevos ojos" permitirán seguir avanzando. 


\section{REFERENCIAS}

AGUILAR, María Constanza y RENTERÍA, Érico (Comp.). Psicología del trabajo y de las organizaciones. Reflexiones y experiencias de investigación. Bogotá, D. C.: Universidad Santo Tomás y Universidad del Valle, 2009. p. 27-51. 697 p.

ARANCIBIA M., Joselyn y MARCHANT R., Loreto. Clima Organizacional como herramienta de gestión. En: MARCHANT R., Loreto (Comp.). Actualizaciones para el management y el desarrollo Organizacional. Valparaíso: Universidad de Viña del Mar, 2007. p. 133-142.

ATEHORTÚA HURTADO, Federico. Gestión y auditoría de la calidad para organizaciones públicas. Norma NTCGP 1000: 2004 conforme a la Ley 872 de 2003. Medellín: Universidad de Antioquia, 2005. $246 \mathrm{p}$.

BABATIVA RODRÍGUEZ, Olga Lucía y HERNÁNDEZ SABOGAL, Marcela. Determinación y evaluación de la gestión del conocimiento en una organización del sector farmacéutico nacional. En: Signos. Investigación en Sistemas de Gestión. 2011, vol. 1, n. 1 y 2, p. 39-60.

BUSTAMANTE VÉLEZ, Ramón Elías. Gestión humana basada en competencias: un modelo integrado a los sistemas de gestión de calidad para el sector público colombiano. En: Revista Interamericana de Psicología Ocupacional. 2006, vol. 25, n. ${ }^{0} 2$, p. 106-123. Consultado el 25 de julio de 2012. Disponible en Internet: http://www.cincel. com.co/rg_25_2.php.

CAMISÓN ZORNOZA, César; CRUZ ROS, Sonia y GONZÁLEZ CRUZ, Tomás. Gestión de la calidad. Conceptos, enfoques, modelos y sistemas. Madrid: Pearson Prentice Hall, 2008. 1428 p.
CUATRECASAS ARBOS, Luis. Gestión integral de la calidad. Implantación, control y certificación. Barcelona: Gestión 2000, 1999. 348 p.

DIETER SIEGEL, Hans. ISO 9000: La llave de entrada al mercado internacional. En: Decisiones Empresariales. Soluciones para Pequeña y Mediana Empresa. 1997, n. ${ }^{0}$ 17, p. 29-31.

ENCISO, Eleonora y PERILLA, Lyria Esperanza. Visión retrospectiva, actual y prospectiva de la psicología organizacional. En: Acta Colombiana de Psicología. 2004, n. ${ }^{0}$ 11, p. 5-22.

GALVIS, Carlos Miguel. Desarrollo de la psicología organizacional y el entorno laboral contemporáneo. En: Cuadernos de Psicología. 2007, vol. 3, n.o 2, p. 11-22.

GONZÁLEZ, Tito. Dificultades en la certificación de calidad normas ISO. Buenos Aires: El Cid Editor, 2009. 27 p.

HAY GROUP. Las competencias como herramienta para identificar individuos con alto desempeño: una visión general. En: Working Paper, 2004a, p. 1-15. Consultado el 20 de abril de 2012. Disponible en Internet: https://ctp.uniandes.edu.co/ Documentos/Las_Competencias.pdf.

Las competencias: clave para una gestión integrada de los recursos humanos. Barcelona: Deusto, 2004b. 189 p.

INSTITUTO COLOMBIANO DE NORMAS TÉGNICAS Y CERTIFICACIÓN (ICONTEC). Gestión para el éxito sostenido de una organización. Enfoque de gestión de la calidad. NTC-ISO 9004. Bogotá, D. C.: ICONTEC, 2010. 52 p. 
. Normas fundamentales sobre la gestión de la calidad y documentos de orientación para su aplicación. Bogotá, D.C.: ICONTEC, 2004. 293 p.

Sistema de gestión de la calidad. Fundamento y vocabulario. NTG-ISO 9000:2000. Bogotá, D. C.: ICONTEC, 2005. 41 p.

- Sistemas de gestión de la calidad. Requisitos. NTC-ISO 9001:2008. Bogotá, D. C.: ICONTEC, 2008. 35 p.

KRIEGEL, Robert y BRANDT, David. De las vacas sagradas se hacen las mejores hamburguesas. Estrategias rompeparadigmas para desarrollar personas y organizaciones dispuestas al cambio. Bogotá, D.C.: Norma, 2006. 392 p.

LÓPEZ REY, Susana. Sistemas de calidad. Implantación de diferentes sistemas en la organización. Bogotá, D.C.: Ediciones de la U, 2011. 166 p.

MAGD, Hesham. An Investigation of ISO 9000 adoption in Saudi Arabia. En: Managerial Auditing Journal, 2006, vol. 21, n. ${ }^{0}$ 2, p. 132-147. Consultado el 23 de enero de 2013. Disponible en Internet: http://www.qualityfoundation.in/downloads/ wbut/7-An_investiga tion\%20-audi\%20arabia.pdf.

MÉNDEZ ÁLVAREZ, Carlos Eduardo. Así somos... ¿Y qué? Cuatro relatos de cultura en gestión empresarial. Bogotá, D.C.: El Autor, 2009.

MÉNDEZ GARCÍA, José C., JARAMILLO VIGUERAS, David, y SERRANO CRESPO, Ildefonso. Gestión de la calidad en procesos de servicios y productivos. México, D. F.: Instituto Politécnico Nacional, 2009. 236 p.

MUJICA DE GONZÁLEZ, Mariálida y PÉREZ DE MALDONADO, Isabel. Gestión del clima organizacional: Una acción deseable en la
Universidad. En: Laurus. 2007, vol. 13, n. ${ }^{0}$ 24, p. 290-304. Consultado el 26 de enero de 2013. Disponible en Internet: http://redalyc.uaemex.mx/ redalyc/pdf/761/76111485014.pdf.

PEREDA MARÍN, Santiago y BERROCAL, Francisca. Gestión de recursos humanos por competencias. Madrid: Centro de Estudios Ramón Areces, S. A., 1999. 276 p.

RAMÍREZ GONZÁLEZ, Margarita. La gestión del conocimiento y su relación con la gestión de la calidad en instituciones del sector agropecuario dedicadas a la ciencia y la tecnología. En: Signos. Investigación en Sistemas de Gestión. 2011, vol. 1, n. ${ }^{0} 1$ y 2, p. 15-37.

ROBLEDO, Ángela María. Psicología y mundos laborales contemporáneos. En: Cuadernos de Psicología. 2007, vol. 3, n. ${ }^{0}$ 2, p. 5-9.

RODRÍGUEZ, Carolina, et al. Aproximaciones al concepto de calidad desde la filosofía. En: Revista de la Universidad de La Salle. 2009, n. ${ }^{0}$ 48, p. 225-241.

SARAVIA, Marcelo A. Recursos humanos en el siglo XXI. Gestión de competencias laborales desde un modelo práctico. 2008. Consultado el 15 de abril de 2012. Disponible en Internet: http://www.conacyt.gov.bo/convocatorias/publicaciones/Gestion CompetenciasLaborales.pdf.

SILICEO AGUILAR, Alfonso, CASARES ARRANGOIZ, David, GONZÁLEZ MARTÍNEZ, José Luis. Liderazgo, valores y cultura organizacional. Hacia una organización competitiva. México, D.F.: McGraw Hill Interamericana, 2000. 220 p.

SORACIPA PULIDO, Nancy Yaneth. Definición de estrategias de transformación de la cultura organizacional en función de un sistema de gestión de 
calidad. En: Signos. Investigación en Sistemas de Gestión. 2009, vol. 1, n. ${ }^{0}$ 1, p. 139-151.

TARÍ GUILLÓ, Juan José, MOLINA MANCHÓN, Hipólito, SABATER SEMPERE, Vicente. Barreras potenciales para implantar la norma ISO 9000. En: Alta Dirección. 2003, vol. 39, n. ${ }^{\circ} 229$, p. 197-202.

TEJADA LOSADA, Fabio. El sistema de gestión de la calidad como aporte para el desarrollo de habilidades y capacidad gerencial en las pymes de Bogotá. En: Signos. Investigación en Sistemas de Gestión. 2009, vol. 1, n. ${ }^{\circ}$ 1, p. 33-68.

TEJADA VARGAS, Natalia y TEJADA LOSADA, Fabio. El Problem Solving estratégico, herramienta para gestionar el cambio en las organizaciones. En: Signos. Investigación en Sistemas de Gestión. 2011, vol. 1, n. 1 y 2, p. 173-183.
TOVAR GÓMEZ, Ariel. La implementación de un sistema de gestión de calidad y el fortalecimiento de la cultura de la calidad en la Universidad de La Salle. En: Revista de la Universidad de La Salle. 2009, n. ${ }^{\circ} 48$, p.100-114.

URIBE MACÍAS, Mario Enrique. Los sistemas de gestión de la calidad. El enfoque teórico y la aplicación empresarial. Ibagué: Universidad del Tolima, 2011. 214 p.

VALDERRAMA RUEDA, Ingrith Elizabeth. Sistemas integrados de gestión como herramienta de competitividad. En: Andina Visual. 2009, vol. 4, n. ${ }^{\circ}$ 5, p. 36-38. Consultado el 23 de enero de 2013. Disponible en Internet: http://www.areandina. edu.co/ contenidos/audiovisuales/publiandina/ documentos/revistas/andinavisual/Andina_Visual_Numero_5.pdf. 